\title{
Evaluación de la adaptabilidad de la acacia (Acacia mangium Wild), y bija (Bixa orellana) en áreas degradadas por la actividad minera aluvial en el Chocó biogeográfico, Condoto, Chocó, Colombia
}

\section{Assessing the adaptability of acacia (Acacia mangium Wild), and annatto (Bixa orellana) in degraded areas of alluvial mining in the Chocó Biogeographic, Condoto, Chocó, Colombia}

\author{
Helcías José Ayala Mosquera ${ }^{1,{ }^{*},}$ Jovanny Mosquera Pino ${ }^{2}$, Wiston Iván Murillo Moreno ${ }^{2}$
}

\section{RESUMEN}

Las prácticas mineras en el Chocó biogeográfico han soportado el desarrollo o el sustento económico de muchas comunidades. Con la introducción de la minería mecanizada, el uso de dragas y retroexcavadoras se ha degradado de manera significativa el medio ambiente y los recursos naturales, situación que se evidencia en el deterioro del componente suelo, la contaminación del recurso hídrico, la desaparición de especies nativas, e impactos relacionados con el componente socioeconómico y cultural de las regiones. Todas estas perturbaciones se presentan en los territorios colectivos de comunidades negras, por lo cual se amerita la urgente necesidad de implementar medidas de restauración que garanticen la sostenibilidad socio ambiental de las tierras que se degradan en la perspectiva de conservación de los recursos naturales y la introducción de dinámicas económicas alternativas en las comunidades mineras. En consistencia con lo anterior, se sometieron a pruebas de adaptación cinco especies forestales: Jatropha curcas, Sacha Inchi Plukenetia volubilis, Balso Ochroma piramidales y Vitabosa Mucuna deeringianum, Acacia y Bija en suelos degradados por minería en el territorio colectivo del consejo comunitario mayor de comunidades negras de Condoto, en área de influencia de la comunidad de La Hilaria. A este respecto reportamos los resultados logrados en una primera fase de estudio de las especies que mejor respuesta de adaptación han tenido, la Acacia y la Bija, las cuales han mostrado buen desarrollo fisiológico, morfológico, anclaje radicular, a partir del sistema de siembra adoptado con diferentes tratamientos y diseños experimentales desde la etapa de vivero hasta la evaluación en sitio definitivo.

Palabras clave. Adaptabilidad; Degradación; Impactos ambientales; Minería aluvial; Restauración; Territorios colectivos.

\begin{abstract}
Mining practices in the Chocó have supported the development or the economic livelihood of many communities, with the introduction of mechanized mining, the use of dredgers and backhoes have been significantly degraded the environment and natural resources, a situation that evidence in land component degradation, pollution of water resources, the disappearance of native species, and impacts associated with the socioeconomic and cultural component of the regions, all these disturbances occur in the collective territories of black
\end{abstract}

1. Investigador del Instituto de Investigaciones Ambientales del Pacífico (IIAP), Coordinador del proyecto de Investigación Evaluación de Alternativas de Recuperación de Áreas Degradadas por Minería en Territorios Colectivos de Comunidades Negras en el Chocó biogeográfico.e-mail:jhenstar@hotmail.com

2. Investigador contratista del Instituto de Investigaciones Ambientales del Pacífico (IIAP) en el proyecto Evaluación de la Adaptabilidad de Especies Vegetales en Áreas Degradadas por Minería en Territorios Colectivos de Comunidades Negras en el Chocó biogeográfico.

e-mail:jovannymosquerapino@hotmail.com wston1980@hotmail.com jhenstar@hotmail.com

Recibido: agosto 27, 2008 Aceptado: septiembre 10, 2008 


\section{Bioetnia Volumen 5 No 2 (julio-diciembre), 2008}

communities, so it is warrants the urgent need to implement restoration measures to ensure environmental and social sustainability of the lands that are degraded in the perspective of natural resource conservation and the introduction of alternative economic dynamics in mining communities. Consistent with this, were tested five species of forest adaptation: Jatropha curcas, Sacha Inchi Plukenetia volubilis, Balso Ochroma pyramidales and Vitabosa Mucuna deeringianum, Acacia and Bija in soils degraded by mining in the territory greater collective community council community Condoto black, area of influence in the community of La Hilaria, in this regard we report the results achieved in the first phase of study of the species that better adaptive response have, the Bija and Acacia, which have shown good physiological development, morphological, root anchorage, from planting system adopted different treatments and experimental designs from the nursery stage to the final site assessment.

Keywords: Adaptability; Degradation; Environmental impacts; Mining alluvial; Restoration collective territories.

\section{INTRODUCCIÓN}

La concepción de la presente investigación en concordancia con las pretensiones comunitarias consideró el enfoque de restauración bajo las siguientes consideraciones: la integración final de la explotación, el mejoramiento visual y paisajístico de las áreas presionadas, aseguramiento de la estabilidad ecosistemica del suelo, el control de los efectos producidos por las aguas de escorrentía y la reconversión económica de la zona a partir de la introducción de nuevas prácticas productivas. También, conjuntamente con el Consejo Comunitario de Condoto y otros actores institucionales que acompañan el proyecto, se analizó que el éxito de esta intencionalidad en el mediano plazo dependerá de los recursos disponibles, de la metodología seleccionada para cada situación, de la disponibilidad de personal capacitado que acompañe el proceso en todas sus fases y componentes, y en especial que las parcelas no sean nuevamente intervenidas por trabajos mineros posteriores.

La investigación parte del análisis comparativo de tres sitios con características contrastantes (colonizado por especies «agresivas», colonizado por especies heliófilas temporales, vegetación con especies secundarias arbórea y arbustiva) derivadas del proceso extractivo irracional de oro y platino, las cuales están localizadas en puntos geográficos específicos del territorio colectivo beneficiario y de las cuales se obtuvo una línea base diagnóstica de los impactos ambientales ocasionados, lo cual permitió la estructuración del plan de recuperación considerando varios factores y variables de análisis, entre ellos, la finalidad de la recuperación, condiciones del suelo, especies a utilizar con perspectiva de conservación y desarrollo económico, y disponibilidad de áreas a intervenir sin riesgo de presión minera en el largo plazo.

A partir de los resultados de la investigación, en este artículo se presenta una metodología para el establecimiento de dos especies vegetales (Acacia mangium y Bixa orellana) en áreas degradadas por minería, describiendo lineamientos específicos para cada una de las especies adaptadas bajo estas condiciones drásticas de degradación.

\section{MATERIALES Y MÉTODOS}

Selección del área de estudio. El área de estudio se preseleccionó de manera concertada entre líderes del Consejo Comunitario de Condoto y expertos de la Universidad Nacional de Colombia, sede Medellín, de Fundación Las Mojarras y del Instituto de Investigaciones Ambientales del Pacífico (IIAP), quienes evaluaron cuatro sectores (Corregimiento de La Hilaria, Barrio Platinero, vereda Jigualito y vereda El Aguacate) a partir de un análisis interdimensional que permitía otorgar una calificación ponderada a cada cualidad de análisis definida a partir de la pretensión del proyecto, tal como se muestra en la Tabla 1.

Ubicación. El área experimental se encuentra ubicada en Colombia en el Municipio de Condoto, corregimiento de La Hilaria a los $5^{\circ} 06^{\prime} 01^{\prime \prime}$ de latitud(N) y $76^{\circ} 32^{\prime} 44.0^{\prime \prime}$ de longitud, a una altura de $70 \mathrm{msnm}$, a 83 kilómetros de Quibdó la capital del Departamento del Chocó. De acuerdo con datos del antiguo Incora, el municipio posee una extensión superficial de 890 kilómetros cuadrados, ocupando $5 \%$ de la territorialidad del Chocó (Figura 1).

Establecimiento de línea base $\boldsymbol{l}^{1}$. El enfoque desde las dimensiones minera, ambiental, geológica, biótica y sociocultural del trabajo permitió la caracterización de la zona para la ejecución del proyecto, cuyo lote piloto es de aproximadamente $4 \mathrm{Ha}$, perteneciente a un área de relleno con material estéril. El alcance previsto era conocer las condiciones actuales del área de influencia y su entorno socioambiental, con el objeto de determinar las variables de análisis en la implementación de un proyecto comunitario de restauración que integrará aspectos productivos, organizativos que garantizaran la recuperación ambiental y la sustentabilidad de los pobladores locales (rehabilitación funcional del ecosistema y el ambiente).

Componente minero ambiental ${ }^{1}$. Para el análisis ambiental se trabajó con una metodología enfocada en la estimación de los pasivos ambientales generados por la minería, confrontando datos locales y estimando el área general impactada

1. Estudio realizado en Convenio por la Universidad Nacional de Colombia, Sede Medellín, investigadores del IIAP y el acompañamiento de miembros de FUNDAMOJARRAS. 
Tabla 1

Evaluación por lugares preseleccionado

\begin{tabular}{lcccc}
\hline Descripción/sitio & La Hilaria & Platinero & Jigualito & El Aguacate \\
\hline Actividad económica & 3.3 & 2.4 & 4.6 & 1.2 \\
Aspecto sociocultural (identidad) & 4.3 & 2.5 & 4.2 & 3.8 \\
Aspecto demográfico & 3.6 & 4.2 & 2.6 & 1 \\
Aspecto organizacional & 2.0 & 3 & 3.6 & 1.7 \\
$\begin{array}{l}\text { Territorio, apropiación colectiva, defensa } \\
\text { del territorio }\end{array}$ & 2.6 & 2 & 3 & 2 \\
Geobiótico: potencial y deterioro & 4.5 & 2.8 & 2.6 & 2.3 \\
Geológico-minero* & & & & \\
Equipamientos, infraestructura & 4.0 & 4.6 & 2.6 & 1.5 \\
Totales & 24.3 & 21.5 & 23.2 & 13.3 \\
\hline
\end{tabular}

* No se calificó este aspecto por falta de valoración cualificada de la zona por parte del equipo geológico-minero que aún no lo había recorrido

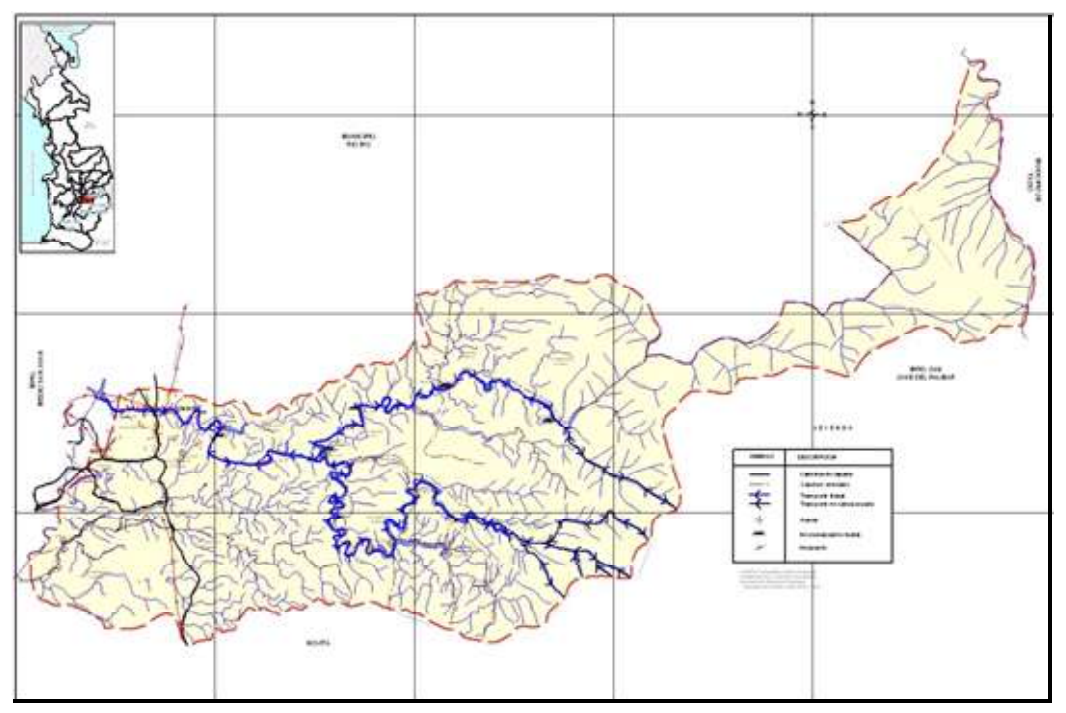

Figura 1. Localización municipio de Condoto

(alrededor de unas 60.000 hectáreas, estimadas a partir de ejercicios de cartografía social realizados con líderes del Consejo Comunitario de Comunidades Negras de Condoto), se recolectaron datos históricos y se realizó una entrevista personal con la dirección técnica de la mina Aluviones de Colombia y dos mineros independientes de la zona, calculando además la capacidad operativa de ambos tipos de explotación.

Componente geológico. La geología de la zona se levantó con base en fuentes secundarias con verificación de campo, que contempló la estimación geológica de yacimientos y la dirección del plano principal superficial del depósito, el cual se georreferenció.

Componente sociocultural. Para dar cuenta de las condiciones culturales, demográficas, económicas y organizativas, de la población y determinar las medidas de trabajo adecuadas, se integraron dos metodologías etnografía e investigación acción participativa. Para la información minera se entrevistaron autoridades, líderes y algunos de los propietarios, con quienes se pudo indagar sobre los efectos de la minería (positivos y negativos) durante su funcionamiento y durante el cierre de mina. Para la información del uso y manejo de otros recursos se entrevistaron adultos mayores, cazadores, pescadores y agricultores.

Se realizaron talleres con el fin de complementar la información de los personajes claves, se efectuó un diagnóstico rápido participativo (DRP), en el cual se reunió la comunidad y se implementaron las técnicas de mapa mentaly la balanza, lo cual, generó los insumos para el análisis posterior y la elaboración de posibles propuestas para el trabajo de productividad y competitividad.

Se efectuaron encuestas contempladas en tres poblaciones diferentes para su realización, la primera de ellas a los mineros ubicados en la zona de estudio, un segundo grupo, corresponde a los propietarios de las tierras donde se realiza o realizó la extracción minera, y un tercer grupo conformado por adultos mayores, cazadores, pescadores y agricultores.

Las observaciones en campo se complementaron con impresiones y percepciones, que permitieron definir las medidas que ma- 


\section{Bioetnia Volumen 5 No 2 (julio-diciembre), 2008}

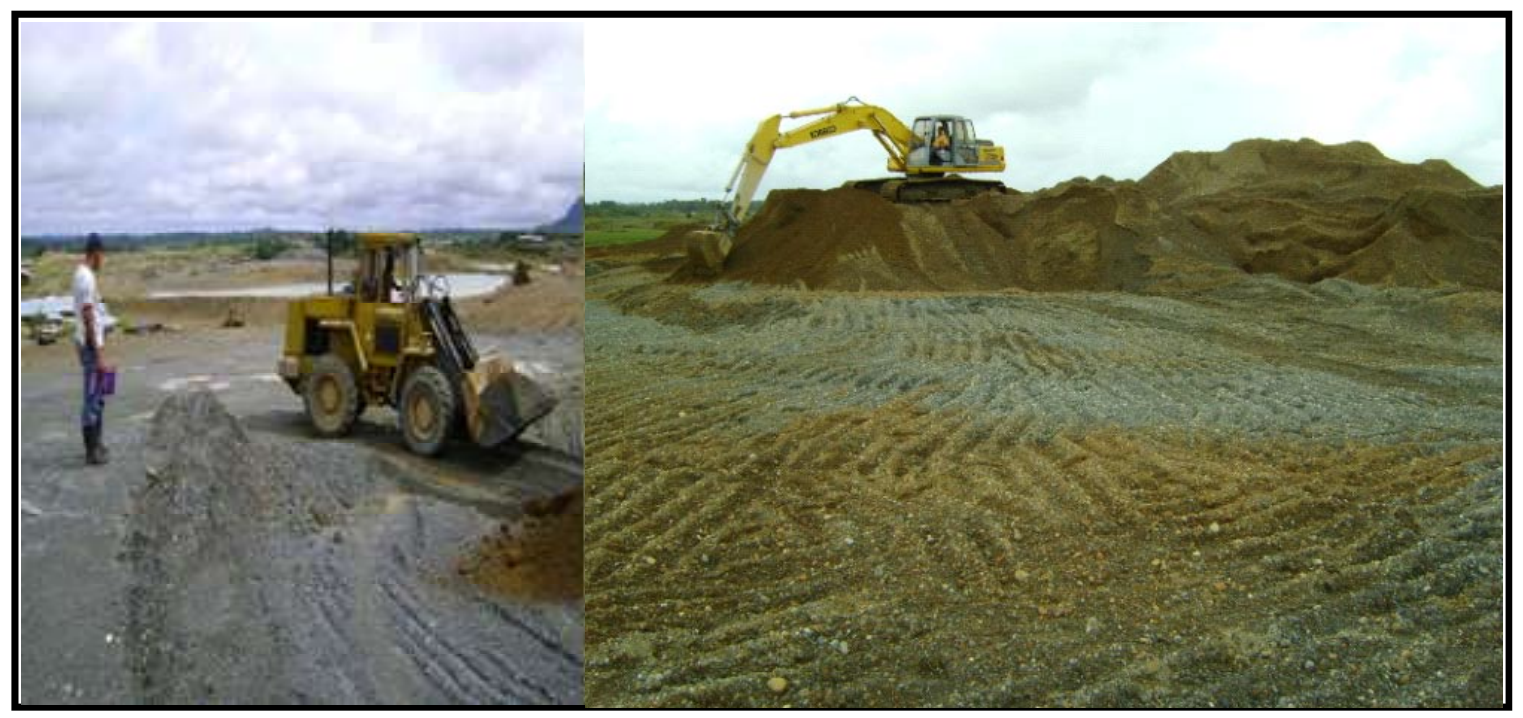

Figura 2. Adecuación delárea experimental

yor impacto positivo tendría sobre la población.

Descripción edáfica ${ }^{2}$. Los suelos fueron analizados en cada una de las zonas seleccionadas tanto para la línea testigo o base (vereda El Aguacate, frente de mina) como para la zona seleccionada para la intervención e implementación del modelo de rehabilitación. El muestreo se realizó de acuerdo con la metodología de la SCSS (Sociedad Colombiana de la Ciencia del Suelo) que contempla un análisis semidetallado de la fertilidad de los suelos con una intensidad de muestreo de 0.3 muestras por hectárea en lotes de manejo similares, configurando 10 submuestras por transecto de $200 \mathrm{~m}^{2}$, aproximando la escala a nivel detallado (11 muestras por hectárea).

La calificación de la fertilidad química se realizó basada en las recomendaciones de la USDA, modificada por ICACORPOICA (séptima aproximación). Los aspectos relacionados con la caracterización del perfil del suelo se analizaron según las recomendaciones de la Soil Survey Staff (color, profundidad, horizontes diagnóstico).

Descripción del recurso hídrico ${ }^{3}$. La calidad del recurso hídrico se estableció tomando muestras compuestas aguas arriba y aguas abajo de las principales fuentes de agua del entorno estudiado, la granja experimental del Consejo Comunitario de Comunidades Negras de Condoto, y el agua residual de la mina mas cercana a la población de La Hilaria, adicionalmente se realizaron aforos de caudal por el método de flotador y se estimó el volumen de sedimentos aportados a la red hídrica local.

2. Análisis realizado por investigadores aliados de la Universidad Nacional, sede Medellín.

3. Se pretendía realizar una valoración diagnóstica para emprender procesos de restauración de sistemas hídricos en perspectiva de motivar la producción piscícola en los módulos o huecos de explotación abandonados y generar alternativas productivas y locales.
Diseño experimental. Se utilizó un diseño experimental de bloques completos al azar con arreglo de parcelas subdividas y tres repeticiones en un área de 1.5 ha en suelos altamente degradados, donde se estableció un vivero transitorio de 32 $\mathrm{m}^{2}$ y se realizaron diferentes tratamientos pre-germinativos a cada una de las dos especies, de tal forma que la investigación se efectuara en dos fases (vivero-sitio definitivo) (Tabla 2).

Para la medición de las especies en vivero se evaluó el \%germinación, \%supervivencia, estado fitosanitario, daño mecánico, altura total de las plántulas, número de foliolos y el crecimiento del fuste; estos datos fueron registrados para cada una de las subparcelas de cada especie y almacenados en la base de datos del proyecto (Tabla 3).

En esta fase se registraron las siguientes variables para cada parcela experimental: altura total de las plántulas, sustrato utilizado en el trasplante, número de foliolos, daño mecánico, estado fitosanitario, crecimiento del fuste, número de individuos sembrados y \% supervivencia de acuerdo con el diseño experimental empleado.

\section{RESULTADOSYDISCUSIONES}

La selección de las especies se basó en el análisis de suelos, el cual arrojó los siguientes resultados:

El análisis de los resultados indican que estos suelos son extremadamente ácidos, no salinos, el perfil modal correspondiente al aguacate presenta altos niveles de saturación por aluminio, bajos a muy bajos niveles de $\mathrm{Ca}, \mathrm{Mg}$ y potasio $(\mathrm{K})$ bajos niveles de fósforo y CIC baja, acordes con la descripción del orden Entisol propuesto.

Sin embargo, es de anotar que los suelos sometidos a proceso de regeneración de la cobertura vegetal, presentan 
Tabla 2

Diseño experimental para la evaluación en vivero

\begin{tabular}{|c|c|c|c|}
\hline Era & Especie & Sustrato & Tratamiento pregerminativo \\
\hline 1 & Acacia mangium & Arena/Gallinaza & $\begin{array}{l}\text { Inmersión en agua hirviendo durante tres minutos y reposo } \\
\text { durante } 12 \text { horas en agua ambiente }\end{array}$ \\
\hline $1-1$ & & Arena/Gallinaza & Inmersión en agua ambiente durante 12 horas \\
\hline $1-2$ & & Arena & Inmersión en agua hirviendo durante tres minutos y reposo \\
\hline $1-3$ & & Gallinaza & $\begin{array}{l}\text { durante } 12 \text { horas en agua ambiente } \\
\text { Inmersión en agua ambiente durante } 12 \text { horas }\end{array}$ \\
\hline 2 & Bixa orellana & Arena/Gallinaza & Sin Tto \\
\hline $2-1$ & & Arena/Gallinaza & Inmersión en agua ambiente durante 6 horas \\
\hline $2-2$ & & Arena & Sin Tto \\
\hline $2-3$ & & Gallinaza & Inmersión en agua ambiente durante 6 horas \\
\hline
\end{tabular}

Observaciones: La relación para cada una de las eras del sustrato utilizado fue de 3:1, arena/gallinaza

Tabla 3

Diseño experimental evaluación en sitio definitivo

\begin{tabular}{lcccc}
\hline Especies & Superficie $\left(\mathbf{m}^{2}\right)$ & Distancia de siembra (m) & $\begin{array}{c}\text { No de individuos } \\
\text { sembrados (plántulas) }\end{array}$ & Combinación \\
\hline Acacia (A1) & 2.500 & $3 \times 3$ & 277 & \\
Acacia (A2) & 2.500 & $4 \times 4$ & 156 & Bija \\
Acacia (A3) & 2.500 & $3 \times 3$ & 277 & Sin Manejo \\
Bija (B1) & 2.500 & $5 \times 5$ & 100 & Acacia \\
Bija (B2) & 2.500 & $4 \times 4$ & 156 & \\
\hline
\end{tabular}

niveles de fósforo y nitrógeno amoniacal más elevados que la media de los suelos de la zona, lo que podría indicar una restauración lenta de la fertilidad natural del recurso suelo. El mejor resultado en función del tiempo se puede observar en la muestra de la parcela en agroforestería análoga del aguacate y la parcela plantada de Acacia mangium cerca de Aluviones de Colombia.

En general se observó que el recurso suelo no está presente en la zona evaluada impactada por minería a cielo abierto y que su proceso de formación es muy lento o no se da (el suelo sometido a análisis no representa 5\% del volumen total del depósito evaluado).

Los métodos utilizados para el análisis de suelos:

Textura: Boyucos; pH: Agua (1:1); Conductividad eléctrica: Extracto de saturación; Materia orgánica: Walkley Black; $\mathrm{Al}$ : KC1 1M; Ca, Mg, K, Na: Acetato de amonio 1M; CICE: Suma de cationes de cambio; S: Fosfato monocálcico 0.008M; Fe, Mn, Cu, Zn:Olsen-EDTA; B: Agua caliente; NO3: Sulfato de aluminio 0.025M; NH4: KC1 1M;P: Bray II.

\section{EVALUACIÓN EN VIVERO}

Acacia. Las semillas fueron sembradas en vivero el 12 de agosto de 2008 e iniciaron a germinar heterogéneamente a los 13 días, presentando hojas compuestas durante los 22 días después de la germinación; luego sus hojas se modificaron a foliolos simples y de bordes enteros, lo que indica que el peciolo simplemente se aplanó.

Se construyeron seis sub-eras de germinación de acuerdo con el tipo de sustrato y se sembraron las semillas en cada una de las cuatro sub-eras basados en el tratamiento pregerminativo para poder determinar cual de los dos tratamientos pregerminativos acelera $\mathrm{y} / \mathrm{o}$ aumenta el porcentaje de germinación en cada uno de los sustratos como lo indica en la Tabla 2 .

Bija o achiote. Las semillas de bija se sembraron en seis sub-eras de germinación cada una con sustratos diferentes, para luego depositar las semillas de acuerdo con el tratamiento pregerminativo que se les realizó. Estas semillas fueron 


\section{Bioetnia Volumen 5 № 2 (julio-diciembre), 2008}
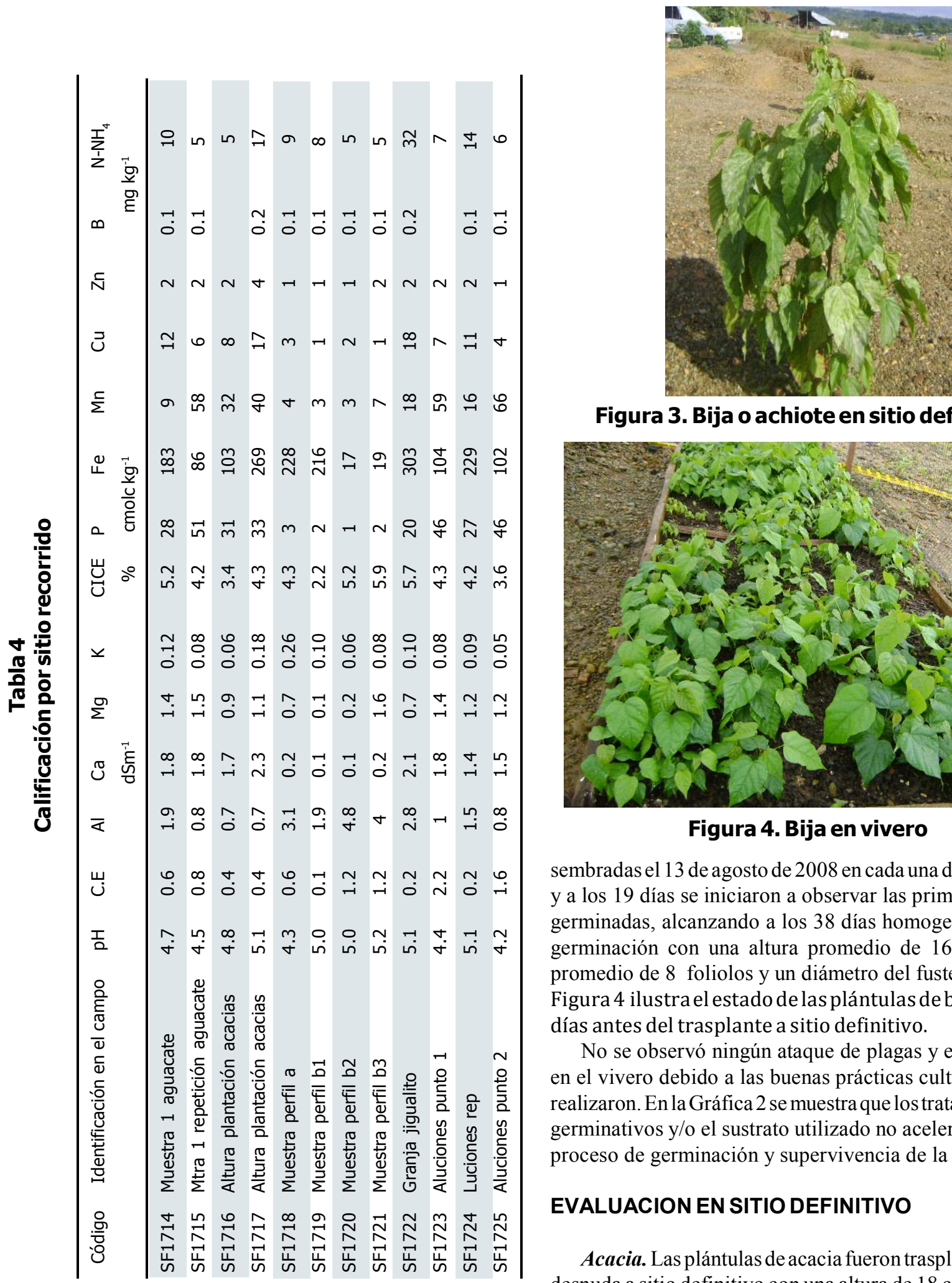

Figura 3. Bija o achiote en sitio definitivo

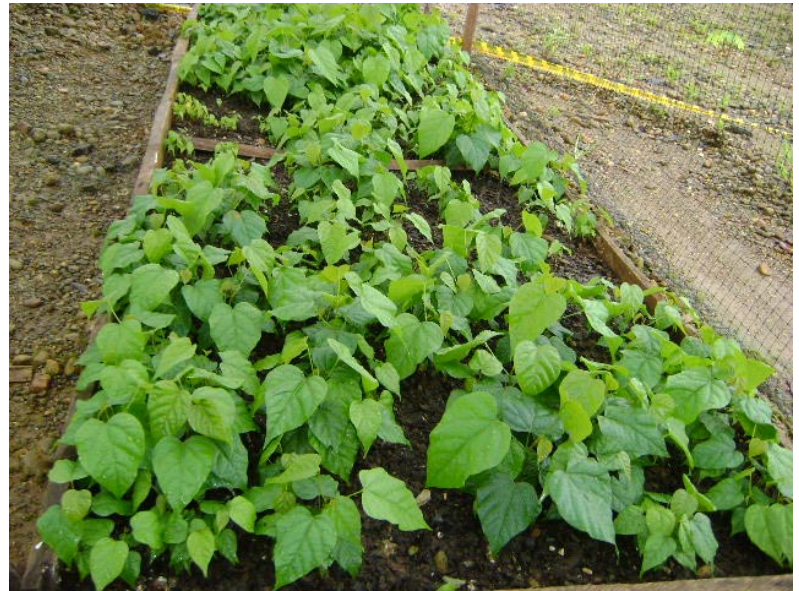

Figura 4. Bija en vivero

sembradas el 13 de agosto de 2008 en cada una de las sub-eras y a los 19 días se iniciaron a observar las primeras semillas germinadas, alcanzando a los 38 días homogeneidad en la germinación con una altura promedio de $16 \mathrm{~cm}$, con un promedio de 8 foliolos y un diámetro del fuste de $2 \mathrm{~cm}$. La Figura 4 ilustra el estado de las plántulas de bija en vivero días antes del trasplante a sitio definitivo.

No se observó ningún ataque de plagas y enfermedades en el vivero debido a las buenas prácticas culturales que se realizaron. En la Gráfica 2 se muestra que los tratamientos pregerminativos y/o el sustrato utilizado no acelera o retrasa el proceso de germinación y supervivencia de la especie.

\section{EVALUACION EN SITIO DEFINITIVO}

Acacia. Las plántulas de acacia fueron trasplantadas a raíz desnuda a sitio definitivo con una altura de $18 \mathrm{~cm}, 5$ foliolos, $3.6 \mathrm{~cm}$ de diámetro del tallo, sin bifurcaciones y en buen estado fitosanitario; para el ahoyado se depositó una planta por 
hoyo y utilizando un sustrato compuesto por gallinaza-arena en relación 3:1 en un área de media ha subdividida en dos subparcelas de un cuarto de ha cada una. Se identifico una tercera subparcela de un cuarto de ha establecida por personal de la zona como parcela testigo.

En la primera subparcela de acacia $\mathrm{N}^{\circ} 1$ (A1), sembrada a una distancia de siembra de 3 metros entre plantas y 3 metros entre surcos, se obtuvo un porcentaje de supervivencia de $100 \%$, es decir, de 277 plantas sembradas, 277 plantas se adaptaron bien al sistema sin presentar volcamientos ni bifurcaciones (Figura 5).

La subparcela $\mathrm{N}^{\circ} 2$ (A2), sembrada en asocio con bija a una distancia de siembra de $4 \mathrm{mx} 4 \mathrm{~m}$, tuvo un porcentaje de $100 \%$. De las 156 plántulas que se sembraron, 156 árboles viven con una altura total de 1,28 metros y un diámetro del fuste de $4,6 \mathrm{~cm}$ sin presencia de torceduras en el tronco ni volcamientos (Figura 6).

A las subparcelas A1 y A2 se les efectuaron los siguientes manejo agrosilvicultural: podas de mantenimiento y formación, desyerbe y fertilización foliar con úrea a los treinta días después del trasplante.

En la subparcela $\mathrm{N}^{\circ} 3$ (A3), denomina parcela testigo, no se realizó ningún manejo agrosilvicultural obteniendo $68 \%$ de árboles vivos de 277 que se sembraron; la mortalidad de $32 \%$ de los árboles se debió a la falta de manejo agrosilvicultural teniendo en cuenta que estas parcelas no presentan suelo y los árboles presentan copa frondosa lo que facilita que los fuertes vientos volqué los individuos (Figura 7, Gráfica 3).

La Gráfica 3, muestra que las distancias de siembras utilizadas no afectaron el buen desarrollo de la especie, sino la falta de manejo agrosilvicultural como lo refleja los resultados de la parcela $\mathrm{N}^{\circ} 3$.

Bija o achiote. Las plántulas de bija se sembraron en sitio definitivo a lo 50 días después de haber germinado con una altura promedio de $16 \mathrm{~cm}$, utilizando como sustrato un compuesto de arena-gallinaza en una relación 1:1, depositando una planta por hoyo en ambas parcelas (Figura 7).

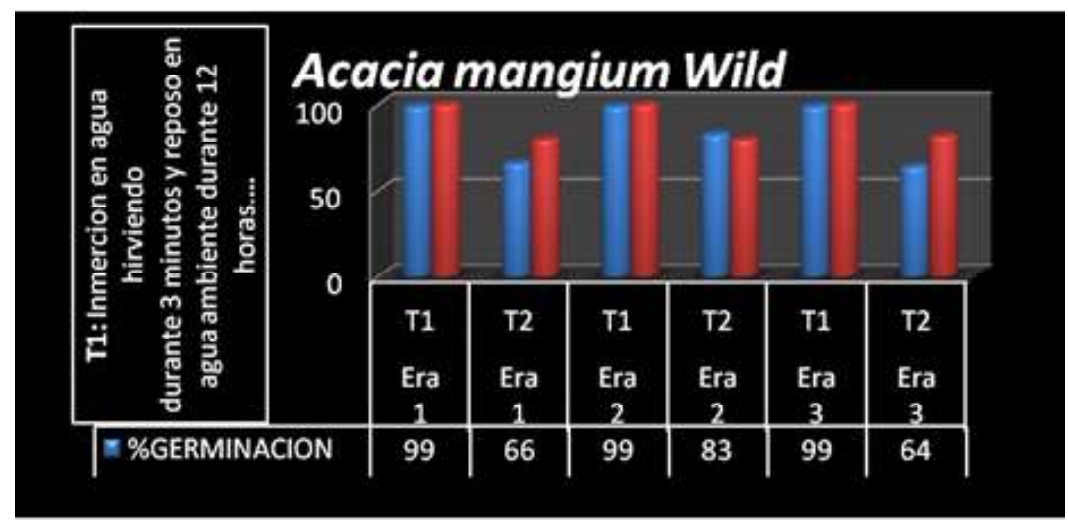

\section{Gráfica 1. Evaluación del porcentaje de germinación y supervivencia de acacia}

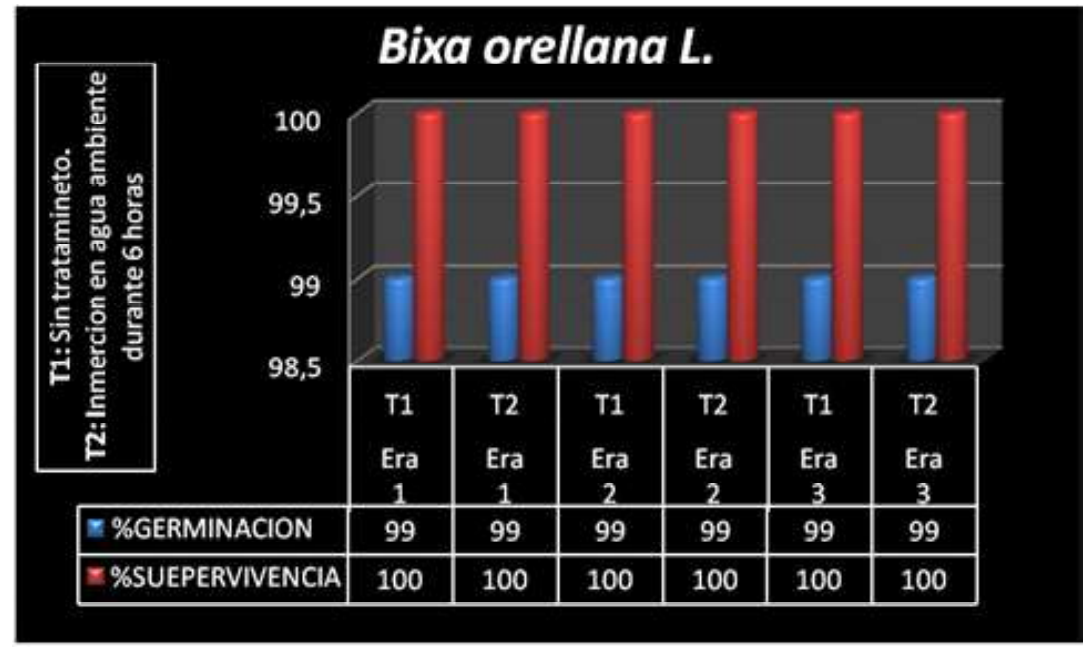

\section{Gráfica 2. Evaluación del porcentaje de germinación y} supervivencia de achiote

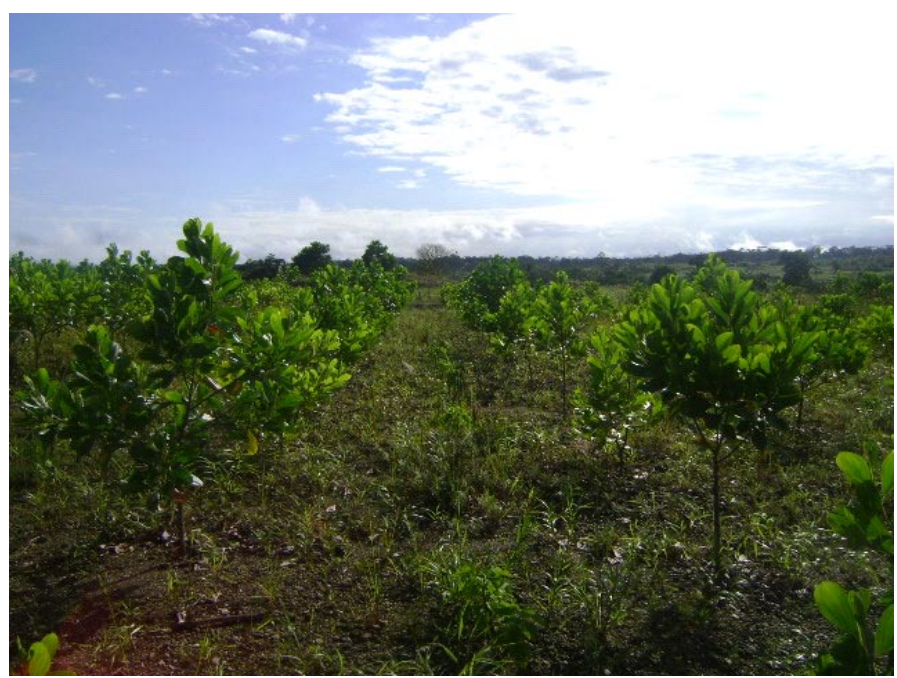

Figura 5. Evaluación de acacia en sitio definitivo parcela A1 


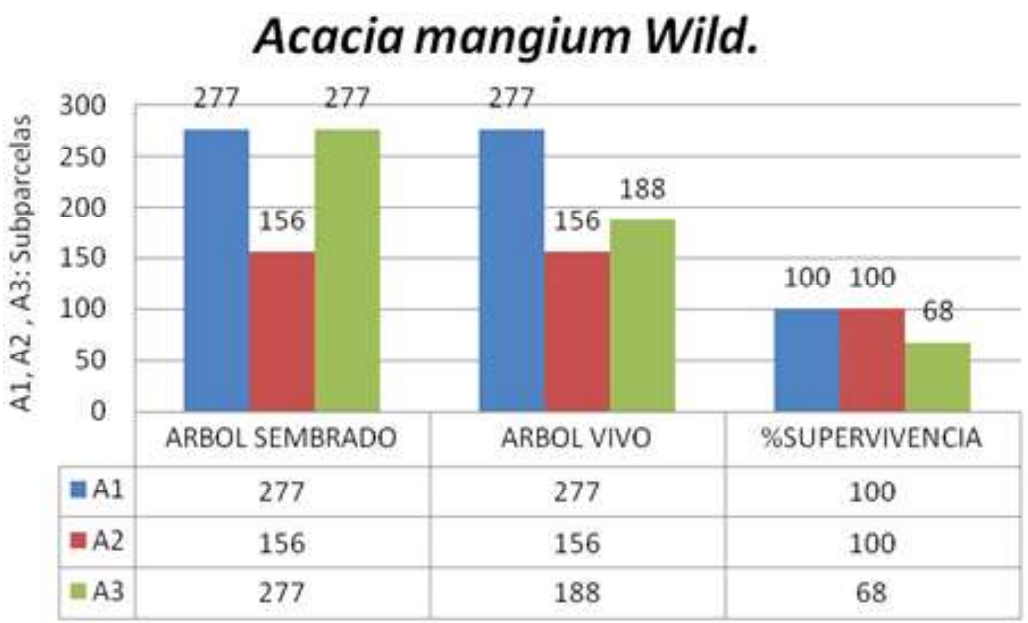

\section{Gráfica 3. Evaluación de la adaptabilidad de acacia en sitio definitivo}

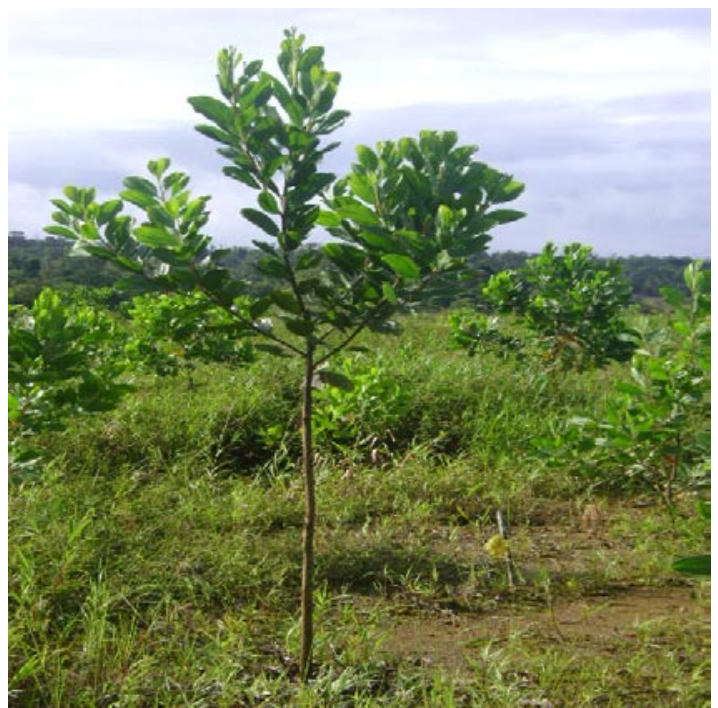

Figura 6. Evaluación de acacia en sitio definitivo parcela $A 2$

\section{CONCLUSIÓN}

Las plántulas de acacia y bija evaluadas en suelos altamente degradados por la actividad minera semi-mecanizada en el corregimiento de La Hilaria, desarrollaron sus partes vegetativas muy bien, lo cual, permite concluir que se adaptan a estos suelos con la aplicación de los tratamientos descritos antes. Esta afirmación se apoya también en los resultados de otras experiencias, que dentro de la presente investigación se documentaron y sistematizaron, identificando ensayos realizados en la zona con condiciones ambientales y edáficas iguales o similares a las presentes en el caso de estudio, donde sin ningún tipo de manejo agrosilvicultural $70 \%$ de de los individuos evaluados de acacia y $40 \%$ de los individuos de

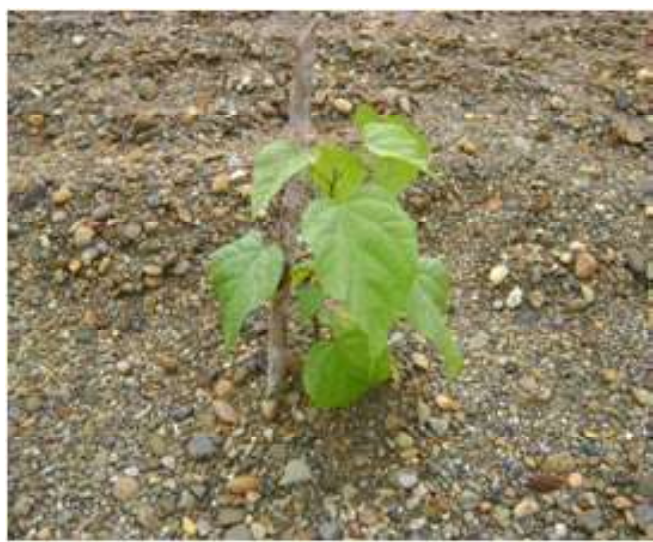

Figura 7. Sistema experimental de acacia y bija

bija, presentan un buen desarrollo vegetativo y reproductivo.

Los individuos de acacia tienen un crecimiento promedio mensual del tallo de $0,2 \mathrm{~cm}$ y una altura total promedio de $1,43 \mathrm{~cm}$. Debido a la buenas prácticas agrosilviculturales que se le realizan, a los individuos de ambas parcelas el 99\% no presenta bifurcaciones a baja altura del tallo y nunca se han volcado en relación con las parcelas A1 y A2.

En ensayos realizados en una parcela aledaña a las del experimento establecido por entables mineros arraigados en la zona de estudio, es común el volcamiento, fundamentalmente por la presencia de vientos muy fuertes y porque en las tierras degradadas por minería no quedan horizontes que le permitan al árbol anclarse debidamente y sólo se sostiene con las raíces laterales.

Si el propósito de la plantación es comercial, se deben podar los árboles, porque estos tienen tendencia a desarrollar ramas gruesas desde muy temprano y a generar más de un fuste; adicionalmente, éstas son condiciones ideales si los propósitos son de recuperación de áreas degradadas porque se incrementa sustancialmente el aporte de biomasa. La acacia tiene poda natural pero tardía, por lo cual, para propósitos comerciales, es recomendable hacer podas de formación.

La Acacia mangium muestra buen crecimiento y adaptación a las condiciones de estos suelos, por lo que se considera que es una especie con buen potencial para usarse en planes de reforestación y restauración teniendo en cuenta que con el establecimiento de acacia se crean condiciones favorables en el suelo para el desarrollo de otras especies.

Es importante considerar que a mayor espaciamiento mayor libertad para que elárbol desarrolle ramas y se bifurque, lo cual es conveniente porque habrá mayor producción de biomasa, lo cual se apreció significativamente en la parcela A3, este principio resulta aplicable siempre y cuando no se trate de plantaciones productoras con fines comerciales en el futuro. 
Las pretensiones de recuperación edafológica, paisajística, y de promoción productiva alternativa de las áreas degradadas por minería, considerando el enfoque agronómico y forestal, se limita por la irregularidad de las condiciones morfológicas en las que quedan los terrenos después de ser explotados, esto genera un costo adicional significativo que se podría obviar si se aplicaran sistemas de explotación racional, que permitiera el restablecimiento morfológico de las terrazas aluviales y la reposición de capa de humus o cubierta superficial que recubre los estratos enriquecidos con metales preciosos. Esta situación será un condicionante para el desarrollo de proyectos de restauración en la zona de Condoto, porque los costos de adecuación de terrenos resultan bastante elevados.

Los resultados obtenidos de la adaptabilidad de estas dos especies vegetales, han sido asimilados por la comunidad de La Hilaria, lo cual se refleja en la disposición permanente para acompañar el desarrollo de la investigación, cuyos resultados aún siendo parciales, ya han comenzado a ser replicados en sectores aledaños a las parcelas demostrativas, se vislumbra entonces una alternativa de rehabilitación del recurso suelo con plantaciones que les permitan obtener bienes y servicios ambientales e ingresos económicos, los cuales mejoraran la calidad de vida de las familias, posibilitando la conformación de grupos asociativos para la producción y comercialización en el mediano plazo de productos derivados de estas dos especies, siempre y cuando las instituciones encargadas del fomento productivo en la zona se involucren en este menester.

La metodología «investigación acción participación» impulsada por el IIAP en el desarrollo del proyecto, no sólo facilitó el intercambio de saberes y la extensión de los resultados de manera práctica hacia la comunidad, sino que propicio ambientes de ocupación del tiempo libre, generación de ingresos a los involucrados, y determinó que la participación comunitaria estuviese integrada bajo condiciones espontáneas equitativas de género y generación, porque siempre estuvieron involucradas de manera significativa las mujeres, y los jóvenes de la comunidad.

\section{AGRADECIMIENTOS}

Agradecemos la participación del Consejo Comunitario Mayor de Condoto, Consejo Comunitario Local de La Hilaria, Alcaldía Municipal de Condoto, Universidad Nacional de Colombia sede Medellín, Asociación de Mineros del Chocó, Corporación Autónoma para el Desarrollo Sostenible del Chocó, Instituto Colombiano Agropecuario, Institución Educativa «Luis Lozano Scipión», Fundación Las Mojarras, Corporación Oro Verde y a los señores Vicente Jiménez, Libardo Gómez y Germán Espinal.

\section{LITERATURA CITADA}

Andrade, H. 1999. Dinámica productiva de sistemas silvopastoriles con Acacia mangium y Eucaliyptus deglupta en el trópico húmedo. Tesis Mag. Sc. CATIE. Turrialba, Costa Rica. 68p.

Brown, R., Foster, R., Gross, E., Liegel, L. 1993. Restauración de ecosistemas perturbados por la pequeña minería en la región de Guayana de Venezuela. Corporación Venezolana de Guayana/USDA. Forest service restoration advisory team. $36 \mathrm{p}$.

Corporación Autónoma Regional para el Desarrollo Sostenible del Chocó. 2000. Caracterización de las aguas y afluentes del rio San Juan afectados por la minería, en los municipios de Condoto, Istmina y Tadó. Quibdó-Chocó. Quidbó: CORCHOCÓ.

Corporación Oro Verde. 2004. Estudio para promover la recuperación de áreas degradadas porminería en el municipio de Condoto y el área de influencia del alto San Juan. Quibdó-Chocó. Quidbó: Corporación Oro Verde.

ECO.ENCOS. 2000. Minería. Derechos de las comunidades y desarrollo local. Libro de Resumen. Lima: ECO.ENCOS.

Instituto de Investigaciones Ambientales del Pacifico. 2005. Diagnóstico situacional de la minería artesanal y en pequeña escala desarrollada por afrocolombianos en territorios colectivos de comunidades negras en el Chocó biogeográfico. Región del Pacifico colombiano, Quibdó- Chocó. Quibdó: IIAP.

MADELEÑA - 3. Proyecto de diseminación del cultivo de árboles de uso múltiple en América Central y Panamá. 1992. Acacia mangium Willd. Especie de árbol de uso multiple en América Central. CATIE, Turrialba, Costa Rica.

Torres, Y. 2006. Uso, manejo y aprovechamiento de la biodiversidad. Documento compilado. Quibdó: IIAP.

Ramírez, N. 1997. Biología reproductiva y selección de especies nativas para la recuperación de áreas degradadas; método y significado. Acta Bot Venez. 20 (1): 43-66.

Sánchez, Y., Mosquera, D. 2007. Evaluación del efecto generado por la actividad minera sobre la comunidad de macroinvertebrados acuáticos en el municipio de Tadó, Chocó. Facultad de Ciencias Básicas, Programa de Biología con Énfasis en Recursos Naturales. Trabajo de grado. Quibdó, Chocó. 